\title{
Capillary Zone Electrophoresis-Top-Down Tandem Mass Spectrom- etry for in-depth Characterization of Hemoglobin Proteoforms in Clinical and Veterinary Samples
}

\author{
Alexander Stolz, Ylva Hedeland, Liesa Salzer ${ }^{\dagger}$, Jennifer Römer, Reidun Heiene ${ }^{\dagger \dagger}$, Laurent Leclercq, \\ Hervé Cottet, Jonas Bergquist and Christian Neusüß*
}

Department of Chemistry, Aalen University, Beethovenstraße 1, 73430 Aalen; Germany

ABSTRACT: Hemoglobin $(\mathrm{Hb})$ constitutes an important protein in clinical diagnostics - both in humans and animals. Among the high number of sequence variants, some can cause severe diseases. Moreover, chemical modifications such as glycation and carbamylation serve as important biomarkers for conditions such as diabetes and kidney diseases. In clinical routine analysis of glycated $\mathrm{Hb}$, sequence variants or other $\mathrm{Hb}$ proteoforms can cause interference, resulting in wrong quantification results. We present a versatile and flexible capillary zone electrophoresis-mass spectrometry screening method for Hb proteoforms including sequence variants and modified species extracted from dried blood spot (DBS) samples with virtually no sample preparation. High separation power was achieved by application of a 5-layers successive multiple ionic polymer layers-coated capillary, enabling separation of positional isomers of glycated $\alpha$ - and $\beta$-chains on the intact level. Quantification of glycated Hb was in good correlation with the results obtained in a clinical routine method. Identification and characterization of known and unknown proteoforms was performed by fragmentation of intact precursor ions. $\mathrm{N}$-terminal and lysine glycation could be identified on the $\alpha$ - and $\beta$-chain respectively. The versatility of the method was demonstrated by application to dog and cat DBS samples. We discovered a putative new sequence variant of the $\beta$-chain in $\operatorname{dog}(\mathrm{T} 38 \rightarrow \mathrm{A})$. The presented method enables separation, characterization and quantification of intact proteoforms, including positional isomers of glycated species in a single run. Combined with the simple sample preparation, our method represents a valuable tool to be used for deeper characterization of clinical and veterinary samples.

\section{INTRODUCTION}

Human hemoglobin $(\mathrm{Hb})$ represents one of the proteins with the most diverse variation with currently over 1300 identified sequence variants ${ }^{1}$. The presence of such (genetic) variants, also referred to as hemoglobinopathies, may result in severe clinical conditions such as sickle-cell disease, hence representing a major global health burden ${ }^{2}$. Additionally, $\mathrm{Hb}$ can undergo non-enzymatic modification by other blood constituencies, such as by reaction with urea (carbamylation) and glucose (glycation), the latter being widely analyzed in clinical diagnosis. The potential for modified $\mathrm{Hb}$ to be used as biomarker is especially aspired due to its high persistence in blood (3 months on average ${ }^{3}$ ) Carbamylated $\mathrm{Hb}$ has been proposed as biomarker for kidney diseases to differentiate between acute and chronic kidney failure in humans ${ }^{4}$ and animals $^{5,6}$, as well as a marker in hemodialysis therapy ${ }^{7}$. While carbamylation is not yet routinely used for diagnosis and monitoring of kidney diseases, glycated $\mathrm{Hb}$ (HbA1c) has developed to an indispensable analyte for monitoring and diagnosis of diabetes ${ }^{8,9}$. The conduction of large-scale studies and the attempts to harmonize its measurements are emphasizing its clinical value and the necessity for standardization and evaluation of related analytical methods ${ }^{10-12}$.

Determination of $\mathrm{HbA} 1 \mathrm{c}$ is performed using various analytical methods such as cation-exchange chromatography and capillary electrophoresis with optical detection as well as immunological methods ${ }^{13}$. However, numerous studies have demonstrated that $\mathrm{Hb}$ sequence variants or other proteoforms (term used according to Smith and Kelleher ${ }^{14}$ ) can lead to interferences in identification and quantification of $\mathrm{HbA} 1 \mathrm{c}^{15-}$ ${ }^{18}$. Therefore, mass spectrometry (MS) has been considered as an alternative for unambiguous identification and quantification, not only for glycated $\mathrm{Hb}$, but also for $\mathrm{Hb}$ sequence variants. Research has been focused on matrix-assisted laser desorption/ionization-MS ${ }^{19}$ and direct-infusion electrospray ionization-MS ${ }^{20,21}$. These studies demonstrated good correlation between clinically determined $\mathrm{HbA} 1 \mathrm{c}$ values and individually glycated $\alpha$ - and $\beta$-chains. However, the simultaneous introduction of various $\mathrm{Hb}$ proteoforms together with salts and other blood constituencies into the MS represents a major problem in terms of ion suppression and spectral overlapping of proteoforms, potentially resulting in poor sensitivity and incorrect relative quantification. To reduce the sample complexity simultaneously introduced into the MS, sophisticated separation methods for intact proteins such as capillary zone electrophoresis (CZE) are aspired. Only a few studies applying $\mathrm{CZE}-\mathrm{MS}$ of $\mathrm{Hb}$ (and related major blood proteins) have been published $^{22-24}$. Redman et al. impressively demonstrated the capabilities of CZE-MS performed on a microfluidic chip ${ }^{24}$. They were able to separate glycated $\alpha$ - and $\beta-\mathrm{Hb}$ from the unmodified chains in less than two minutes. To achieve such a high separation power for intact proteins, capillary coatings are needed to reduce interactions between analytes and the capillary wall. Different coating strategies have been developed and their application for CZE-MS has been reviewed ${ }^{25}$. 5-layers successive multiple ionic-polymer layers (SMIL) represent an attractive coating due to the easy preparation, 
high coating stability and possibilities of regeneration ${ }^{26,27}$. These coatings allow effective reduction of analyte-wall interaction as well as fine-control of the electroosmotic flow (EOF), hence enabling adjustment of separation efficiency ${ }^{27}$.

The use of dried blood spots (DBS) is an emerging technique in the clinical context with several advantages such as the low sample amounts needed, low costs and easy handling, storage and shipping ${ }^{28}$. DBS is routinely used in newborn screening for genetic diseases such as phenylketonuria ${ }^{29}$ and sickle cell disease ${ }^{30}$. The application of DBS samples for $\mathrm{Hb}$ analysis in general has been reviewed, summarizing applications and advantages of the technique ${ }^{30}$.

The aim of this study was to develop a versatile screening method for the joint characterization of various $\mathrm{Hb}$ proteoforms (including both, sequence variants as well as posttranslationally modified species) from clinical human and veterinary DBS samples. Particular emphasis was placed on achieving high separation power for intact proteins, applying top-down MS/MS for reliable proteoform identification and characterization as well as evaluating capabilities for relative quantification of glycated and carbamylated $\mathrm{Hb}$.

\section{EXPERIMENTAL SECTION}

\section{Materials and Reagents}

Ultrapure water was generated by an Ultra Clear UV system (Siemens Water Technologies, USA). 2-propanol (LC-MS grade), formic acid $(\geq 98 \%)$ and acetic acid (Supra) were obtained from Roth (Karlsruhe, Germany). 4-(2-hydroxyethyl)1-piperazineethanesulfonic acid (HEPES), D-(+)-glucose $(99.9 \%)$, potassium cyanate $(>96 \%)$, sodium cyanide $(>97 \%)$ and human $\mathrm{Hb}$ were purchased from Sigma-Aldrich (Steinheim, Germany). Sodium hydroxide (1 M) was obtained from Merck (Darmstadt, Germany). Quarternized diethylaminoethyl dextran (DEAEDq) was obtained from Pharmacia (Uppsala, Sweden) and poly(methacrylic acid) (PMA) was purchased from Sigma-Aldrich (Saint-Quentin Fallavier, France). Fused silica capillaries (50 $\mu \mathrm{m}$ ID, $365 \mu \mathrm{m}$ OD) were purchased from Polymicro Technologies (Phoenix, AZ, USA).

\section{Clinical samples and sample preparation}

Human DBS samples were obtained from Uppsala University Hospital (Sweden). All human samples used where leftovers after the completion of diagnostic tests and were used in accordance with the regional ethical review board of Uppsala (no 2001/367). The samples were chosen to cover the range of interest for HbA1c. In three of the samples, an elevated plasma creatinine value was determined, whereas for three other patients, the electropherograms were classified as untypical, indicating the presence of additional $\mathrm{Hb}$ forms (e.g., fetal $\mathrm{Hb}$ $(\mathrm{HbF})$ ). Cat and dog DBS samples were obtained from AniCura (Oslo, Norway) and from the University Animal Hospital (UDS, Uppsala, Sweden). All samples were residual blood samples from diagnostic tests, collected in accordance with national ethical guidelines in Norway and Sweden. Around $20 \mu \mathrm{L}$ whole blood (in K2 EDTA tubes for human samples, Idexx VetCollect tubes for animal samples) were collected on each spot on a Whatman FTA ${ }^{\mathrm{TM}}$ DMPK-C Card (Sigma-Aldrich, Steinheim, Germany).

DBS samples were prepared by punching out a $3 \mathrm{~mm}$ disk on a Whatman cutting mat. Hb was extracted with $100 \mu \mathrm{L}$ deionized water for $30 \mathrm{~min}$ under thorough shaking. The re- sulting solution was centrifuged and subsequently used for analysis. In vitro glycation was performed according to Bae et al. ${ }^{31}$, in vitro carbamylation according to Weykamp et al. ${ }^{32}$. The detailed protocols for preparation can be found in the supporting information.

\section{Capillary coating}

$50 \mu \mathrm{m}$ ID x $70 \mathrm{~cm}$ 5-layers SMIL-coated capillaries were prepared according to the following procedure (all flushing steps were performed with 2 bar pressure): capillaries were initially flushed for $20 \mathrm{~min}$ with $1 \mathrm{M}$ sodium hydroxide, $5 \mathrm{~min}$ with water and $10 \mathrm{~min}$ with $20 \mathrm{mM}$ HEPES buffer. Then, the capillary was alternatingly flushed for $7 \mathrm{~min}$ with $3 \mathrm{mg} / \mathrm{mL}$ polycation and polyanion solutions with a $3 \mathrm{~min} 20 \mathrm{mM}$ HEPES flush between polycation and polyanion solution. After attachment of the last polycation layer, the capillary was flushed with background electrolyte (BGE) for 10 min. Adaptions of the protocol for the coating of $105 \mathrm{~cm}$ capillaries can be found in the supporting information.

\section{CZE-MS}

CZE-MS was performed on an HP 3DCE electrophoresis instrument from Agilent Technology (Waldbronn, Germany). Polyimide coating was removed from the MS side of the capillary. Prior to each injection, the capillary was flushed for 5 min (1 bar) with BGE (2 M acetic acid (HA)). The sample was hydrodynamically injected (70 cm capillary: 50 mbar, $8 \mathrm{~s} ; 105$ cm capillary: 50 mbar, $10 \mathrm{~s}$ ). Separation was performed by application of $-30 \mathrm{kV}$. The CZE system was either coupled to an Agilent 6550 time-of flight (TOF) or to an Orbitrap Fusion Lumos MS. Coupling to the TOF MS was performed via a G1607A sheath liquid interface from Agilent Technologies with 50:50 2-propanol:water with $0.5 \%$ formic acid (FA) as sheath liquid (SL) and a SL flow of $4 \mu \mathrm{L} / \mathrm{min}$. Capillary voltage was set to $4000 \mathrm{~V}$, fragmentor and skimmer voltage to 175 and $65 \mathrm{~V}$ respectively. The mass range was set to 550-2000 $m / z$ with an acquisition rate of $4 \mathrm{~Hz}$. Coupling with the Orbitrap MS was performed with a prototype sheath-liquid interface, kindly provided by François de l'Escaille, (cp. González-Ruiz et. $\mathrm{al}^{33}$ ) with 80:20 2-propanol:water with $0.5 \% \mathrm{FA}$ and $25 \mu \mathrm{M}$ L-methionine as SL and a SL flow of $1 \mu \mathrm{L} / \mathrm{min}$. Methionine was added as a scavenger to reduce in-source oxidation. The capillary was inserted in the stainless steel needle with a capillary protrusion of $\sim 200 \mu \mathrm{m}$ and a distance of $1.8 \mathrm{~mm}$ to the MS transfer capillary. Spectra were acquired in positive ion mode with a spray voltage of $2900 \mathrm{~V}$ and an ion transfer temperature of $300^{\circ} \mathrm{C}$. For the MS1 method, scan range was $650-2000 \mathrm{~m} / z$ with a resolution of 7500, an AGC target of $1 \mathrm{e}^{6}$ (150 ms maximum injection time) and $7 \mu$ Scans. Detailed method parameters for the $\mathrm{MS}^{2}$ and the pseudo selective reaction monitoring (SRM) method can be found in the supporting information.

\section{Data analysis}

TOF MS data analysis, including charge state deconvolution was performed using Agilent Masshunter Workstation (BioConfirm B.06.00). Orbitrap MS data were analyzed using Freestyle 1.5 and deconvolution was performed with PMI Intact Mass v3.4 (Protein Metrics, Cupertino, CA, USA). In general, extracted ion electropherograms (EIEs) were created by summation of the intensity of the three most abundant charge states for a protein. EIEs were smoothed with default parameters in Agilent Mass Hunter, and with the moving mean 
algorithm (7 data points) in Freestyle. All quantification results were obtained by peak integration of these EIEs. Relative quantification of glycated and carbamylated $\alpha$ - and $\beta-\mathrm{Hb}$ was performed by dividing the peak area of the glycated /carbamylated peak by the sum of peak areas of all identified $\alpha$ - or $\beta$-chains. MS/MS data were analyzed using ProSight Lite v1.4 (Northwestern University, Evanston, IL, USA) after deconvolution of the fragment spectra with the Xtract algorithm embedded in Freestyle. Fragment maps were created by using primary sequences obtained from Uniprot with a fragment tolerance of $5 \mathrm{ppm}$.

\section{RESULTS AND DISCUSSION}

\section{Method development}

To find a suitable separation system, initial CZE-MS experiments were performed with in vitro glycated $\mathrm{Hb}$. Selection of possible polycation/polyanion combinations for SMIL-coated capillaries was based on a previous publication ${ }^{27} .0 .5 \mathrm{M}$ and $2 \mathrm{M}$ HA were tested as BGE. Due to the denaturing conditions, separation of the individual $\alpha$ - and $\beta$-chains was observed in contrast to the separation of the intact $\mathrm{Hb}$ complex usually performed in clinical routine methods. Of the tested SMIL coatings, the combination of DEAEDq and PMA together with $2 \mathrm{M} \mathrm{HA}$ as BGE exhibited baseline separation of $\alpha$ - and $\beta$-chains as well as their glycated forms (Figure 1a) and was hence chosen for our method. Additionally, we were able to separate the carbamylated $\alpha$ - and $\beta$-chain in an in vitro carbamylated sample (Figure 1b). Only the $\alpha$-chain has been identified in its carbamylated form, whereas no $\beta$ carbamylation was identified.

(a) $\times 10^{5}$

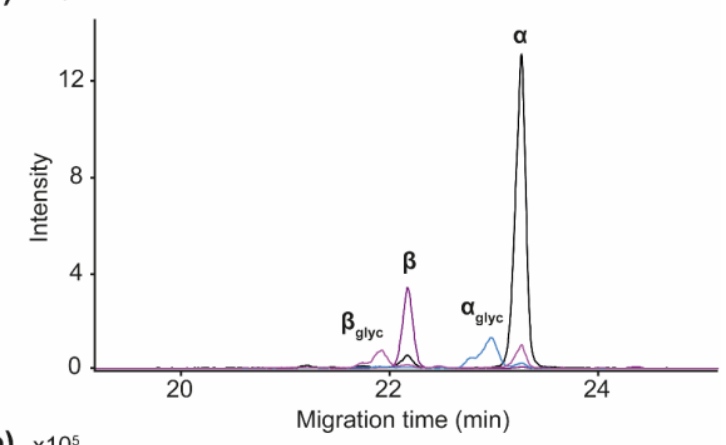

(b)

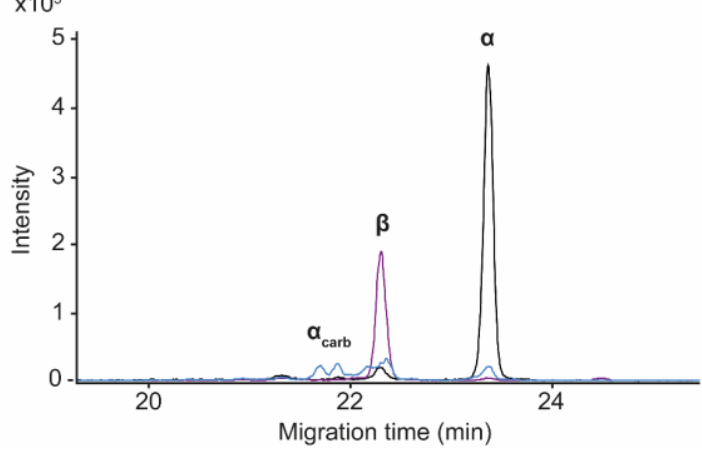

Figure 1. CZE-MS of in vitro glycated and carbamylated Hb. Separation of glycated (a) and carbamylated (b) $\mathrm{Hb}$ was performed in a DEAEDq-PMA coated capillary $(70 \mathrm{~cm})$ with $2 \mathrm{M}$ $\mathrm{HA}$ as BGE and $-30 \mathrm{kV}$ separation voltage coupled to a QTOF MS. EIEs were created by addition of the three most abundant charge states with a mass tolerance of $0.15 \mathrm{~m} / \mathrm{z}$. Black: $\alpha$, purple: $\beta$, blue: $\quad \alpha_{\text {carb }} / \alpha_{\text {glyc }}, \quad$ pink: $\beta_{\text {glyc. }}$
This indicates that the $\alpha$-chain is the main target for in vitro carbamylation.

The relatively low magnitude of the EOF $\left(\mu_{\mathrm{EOF}}\right)$ for this coating in combination with $2 \mathrm{M}$ HA results in low values for the effective mobility for the separated $\mathrm{Hb}$-chains, increasing their relative difference and hence selectivity. Interestingly, both, glycated and carbamylated $\alpha$ - and $\beta-\mathrm{Hb}$ showed a double peak. We attribute this to the presence of diverse glycation and carbamylation sites, resulting in slightly different mobilities for the modified chains, indicating high selectivity of the method. Identification of proteoforms was based on deconvolution of respective mass spectra with subsequent assignment based on intact masses.

SMIL coatings offer an easy and relatively fast coating procedure. Polycation, construction buffer and polyanion solutions merely need to be flushed through the capillary. After approx. 1-2 $\mathrm{h}$ the capillary can be used. We observed acceptable coating stability over multiple measurements. Additionally, simple recoating of the top layer usually provides full recovery of initial coating quality.

\section{Clinical human DBS samples}

After initial method development on a QTOF MS instrument, all subsequent experiments were performed on an Orbitrap MS. Hb was extracted from clinical DBS samples of nine individual anonymous patients. Figure 2 shows representative EIEs for one of the patient's samples. Compared to the QTOF MS data, we observed slightly reduced separation efficiency as can be seen by broader peaks and the absence of the double peaks for

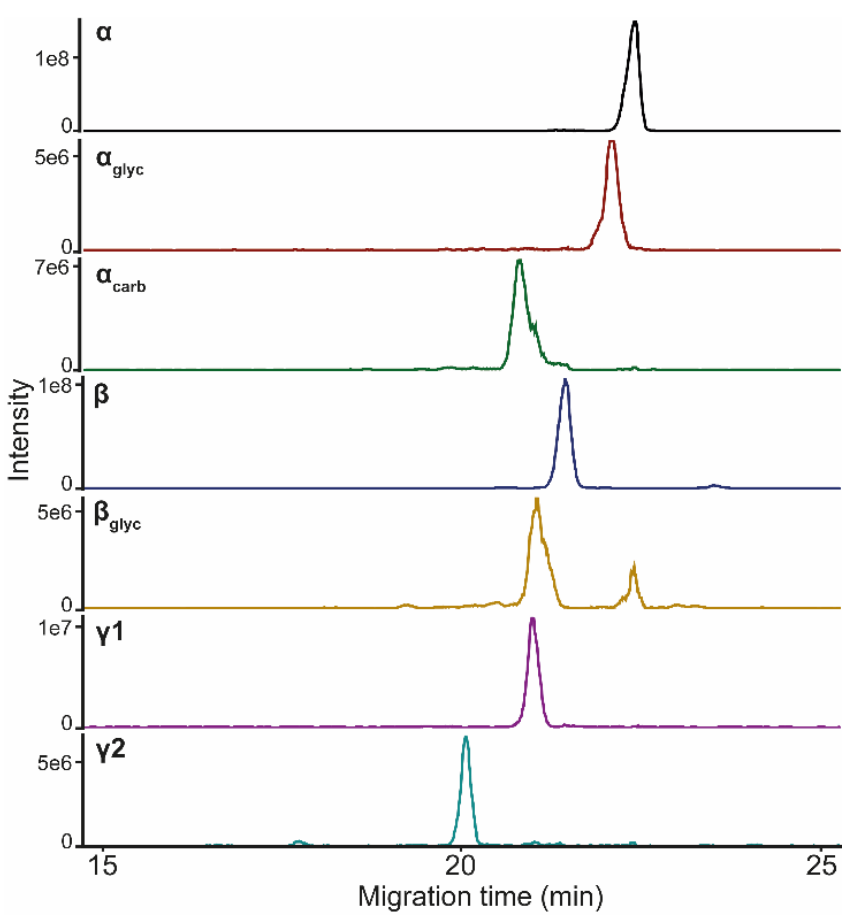

Figure 2. Separation of various $\mathrm{Hb}$-chains from a representative human DBS samples. CZE was performed as previously described and was coupled to an Orbitrap MS. $2^{\text {nd }}$ peak in $\beta_{\text {glyc }}$ EIE results from spectral interference with $\alpha$. EIEs were created by addition of the three most abundant charge states with mass tolerance of $0.15 \mathrm{~m} / \mathrm{z}$. 
glycated $\alpha$ - and $\beta$-chains (cp. Figure 1). We attribute this to the different CZE-MS interfaces used for QTOF and Orbitrap MS. Besides detection of unmodified and glycated $\alpha$ - and $\beta$ $\mathrm{Hb}$, car-bamylated $\alpha-\mathrm{Hb}$ was identified at low abundance. The $\gamma 1$ and $\gamma 2 \mathrm{Hb}$-chains exhibited high abundance $(\sim 10 \% \gamma 1+\gamma 2$ of overall $\beta+\gamma$ chains), compared to average values of $<1 \%$ $\mathrm{HbF}$ in healthy adults, indicating a condition referred to as hereditary persistence of fetal hemoglobin ${ }^{34}$. This result coincides with the detection of an unknown peak in the clinical routine analysis of the sample that has been suspected to contain $\mathrm{HbF}$. Furthermore, $\delta-\mathrm{Hb}$ was identified in all samples which is part of the minor HbA2 form (data not shown).

These results show the applicability of the CZE-MS method for $\mathrm{Hb}$ analysis from DBS samples. We were able to separate and identify the main $\alpha$ - and $\beta$-chain together with minor proteoforms including glycated and carbamylated $\mathrm{Hb}$ as well as $\gamma$ - and $\delta$-chains. The analysis of DBS samples offers easy sample handling and allows extended storage times of samples compared to whole blood ${ }^{35}$. Combined with the analysis of intact $\mathrm{Hb}$-chains rather than proteolytic peptides, sample prep-

(a)
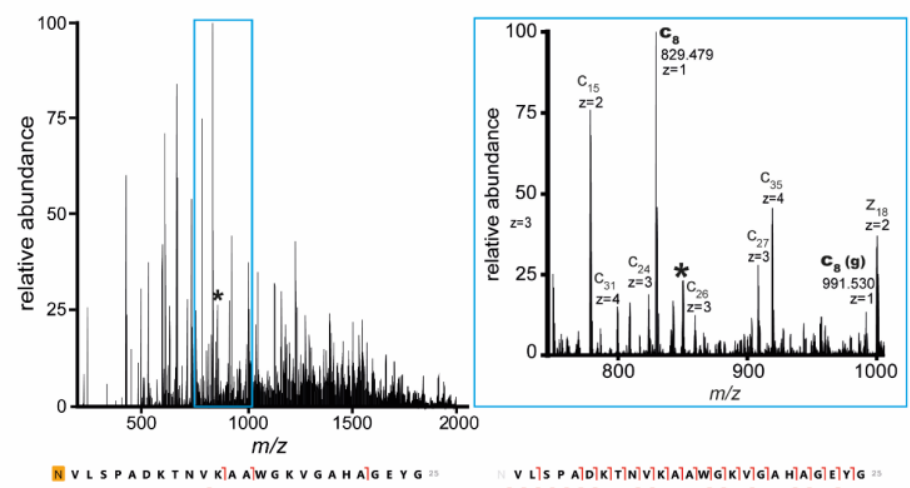

AEALERMFLSTFPTTKTYFPHFDLSH GSAQVKGHIGKKVADALTNAVAHVDD M SIH

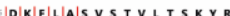

FC: $19 \%$; P-Score: $1.3 e^{-23}$

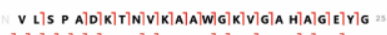

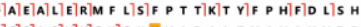

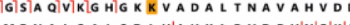
TSIH S LDKKLFLLLALS V S T V L T S K Y R

FC: $45 \%$; P-Score: $9.2 e^{-80}$

(b)
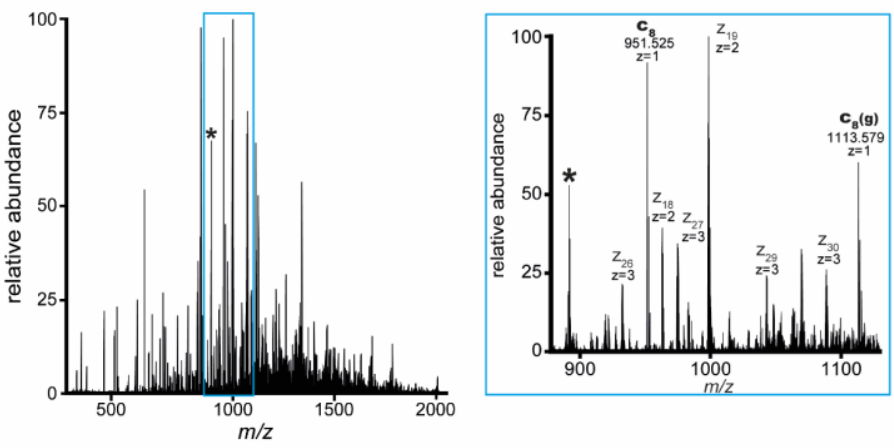

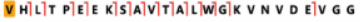
EALG[R]LILTVIVYPIWTQRFFE]SFGDLST PDAVMGNPKV]K[A H]G]KKVLLGA]F SD]GL? A HILLDIN L K GITTFIA T L S E L H C DKL LH VIDP EIN FIRILLIG|N VILV V VILA|HIHIFIG|K|E|F|TP P

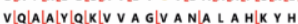

FC: $41 \%$; P-Score: $2.2 \mathrm{e}^{-60}$ aration is fast and simple and the possibility of artificial artifact formation, usually encountered during proteolytic digestion $^{36-38}$, can be reduced.

\section{Top-Down analysis of $\mathbf{H b}$ proteoforms}

Top-down MS/MS was applied for in-depth characterization of glycation and carbamylation sites of modified $\mathrm{Hb}$, as well as for the identification of unknown species. Glycation and carbamylation are reactions on free amino-groups of the protein. This can either be the free $\mathrm{N}$-terminus or any of the 11 ( $\alpha$ and $\beta$ respectively) lysine residues within the protein sequence. Targeted precursor selection of in vitro glycated and carbamylated $\alpha$ - and $\beta-\mathrm{Hb}$ was performed respectively Figure $3 \mathrm{a}$ and $3 \mathrm{~b}$ show representative ETD spectra of glycated $\alpha$ - and $\beta$-Hb. Additionally, fragment maps for $\mathrm{N}$-terminal and lysine glycation are given. Placing the modification on different residues resulted in different fragmentation coverages and $\mathrm{P}$ scores. Estimation of the modification site was based on high fragmentation coverage

(c)

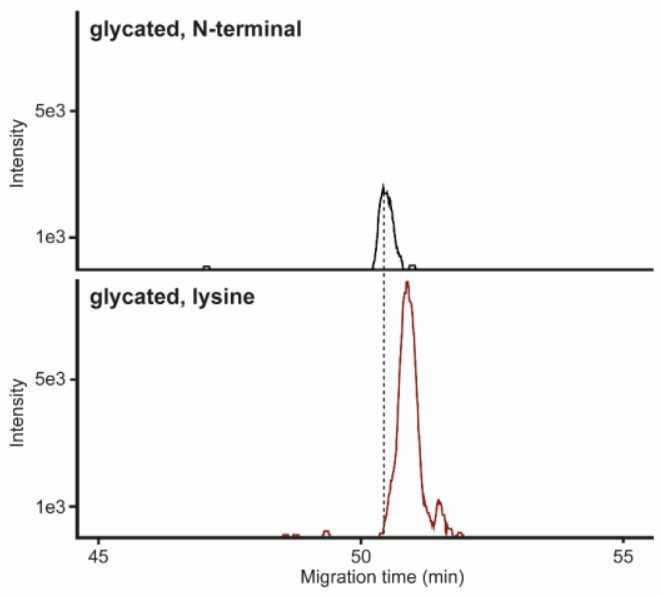

(d)

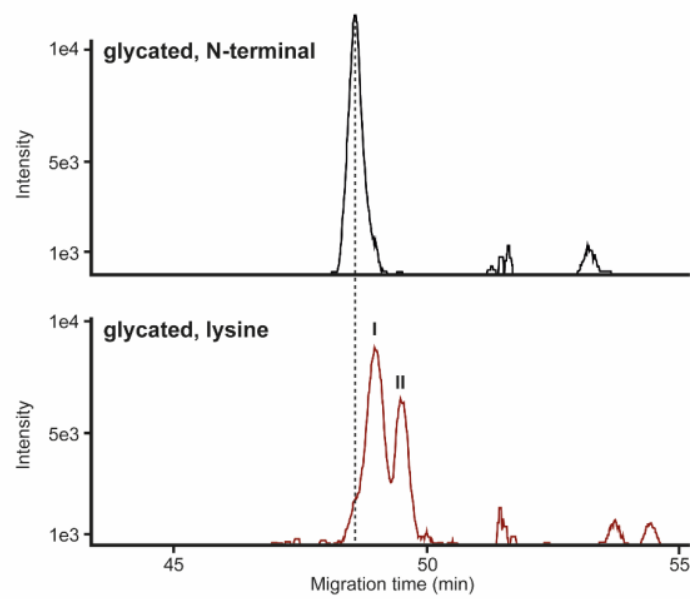

Figure 3. Top-down analysis of in vitro glycated $\mathrm{Hb}$. ETD spectrum of glycated $\alpha-\mathrm{Hb}$ (a) and $\beta-\mathrm{Hb}$ (b). The blue rectangle marks the part of the spectrum containing the diagnostic glycated and non-glycated c8 ion used for the pseudo SRM method. The asterisk represents the precursor ion. Below the spectrum, fragment maps are shown, with orange boxes marking the modification site. Separation of N-terminaland not lysine-glycated $\alpha-\mathrm{Hb}$ (c) and $\beta-\mathrm{Hb}$ (d). Separation was performed on a $105 \mathrm{~cm}$ DEAEDq-PMA coated capillary as previously described. FC: Fragmentation coverage. 
and low P-scores. For both chains, c-ion series could be identified for $\mathrm{N}$-terminal- and lysine-glycated species. Especially for the $\beta$-chain, the ion series resulted in comparable fragmentation coverage (41\% N-terminal vs. $43 \%$ lysine glycation), indicating the presence of at least two positional isomers that are co-isolated, resulting in a chimeric MS/MS spectra.

For the $\alpha$-chain, fragmentation coverage was considerably higher when placing the modification on certain lysine residues of the protein, compared to the N-terminal positioning, indicating low abundance of the N-terminal-glycated chain. Different spectral averaging across the peak of glycated $\beta-\mathrm{Hb}$ showed varying fragmentation coverages and $\mathrm{P}$ - scores for $\mathrm{N}$ terminal or lysine glycation, indicating partial separation of the positional isomers as postulated in CZE-QTOF MS measurements (cp. Figure 1). To verify this assumption, a pseudo SRM method was developed by tracing diagnostic ions resulting from ETD fragmentation. Figure $3 a$ and $3 b$ show an enlarged part of the MS/MS spectra with the diagnostic $c_{8}$ ions used for the pseudo SRM method. Co-selection of N-terminaland lysine-glycated $\alpha$ - or $\beta$-chains leads to the formation of two different $c_{8}$ ions, one with attached glycation, one without. Therefore, these transitions can be used to visualize the separation of the positional isomers. Figure $3 \mathrm{c}$ shows the separation of N-terminal- and lysine-glycated $\alpha-\mathrm{Hb}$ in a $105 \mathrm{~cm}$ DEAEDq-PMA coated capillary. A longer capillary was used to enhance separation of isomers, allowing deeper characterization of modification sites. Since the same precursor is used for both species, relative quantification can be performed by comparison of peak areas. $19 \%$ of the glycated $\alpha-\mathrm{Hb}$ was $\mathrm{N}$ terminal modified while the major proportion (81\%) was glycated on lysine residues. For the $\beta$-chain (Figure $3 \mathrm{~d}$ ), a higher proportion of $\mathrm{N}$-terminal glycation could be identified $(40 \%)$. Interestingly, the EIE of the lysine-glycated $\beta$-chain shows a double peak, suggesting the presence of at least two different lysine-glycated species (40\% and 20\% respectively). We suspect that the peaks of the lysine-glycated species (for $\alpha$ and $\beta$ ) consists of multiple overlapping positional isomers. We could find ion series matching multiple glycation sites. Highest fragmentation coverage and lowest P-score was observed when placing the modification on K60 or K61 for $\alpha$ and K59 for $\beta$, indicating that these represent major modification sites in this mixture.

As shown in previous results, carbamylation was only detected on the $\alpha$-chain. Similarly to the glycated species, at the front of the CZE-peak, fragmentation coverage was highest when putting the modification on $\mathrm{N}$-terminal position, whereas the tail of the peak reveals internal, lysine carbamylation (data not shown). As for the glycated chain, the exact location of modification for the lysine-carbamylated species could not been pinpointed.

By application of pseudo SRM we were able to confirm partial separation of positional isomers of intact glycated and carbamylated Hb-chains. This impressively shows the high separation power of CZE using DEAEDq-PMA coated capillaries.

\section{Quantification of modified $\mathrm{Hb}$}

Our CZE-MS method allows relative quantification of modified Hb-chains, whereas the pseudo SRM method allows quantification of positional isomers. These results are summarized in Figure 4. Figure 4a shows quantification results for glycated and carbamylated $\mathrm{Hb}$ for nine clinical DBS samples.
Patient 1 shows significantly increased values for glycated $\alpha$ and $\beta-\mathrm{Hb}$ which coincides with an elevated clinical HbA1c value. All other values lie within the same range and appear to be logical basal levels for healthy adults. For six of the nine samples, HbA1c values from clinical analysis were available (details can be found in the supporting information). We used these values to evaluate linear correlation between our quantification results

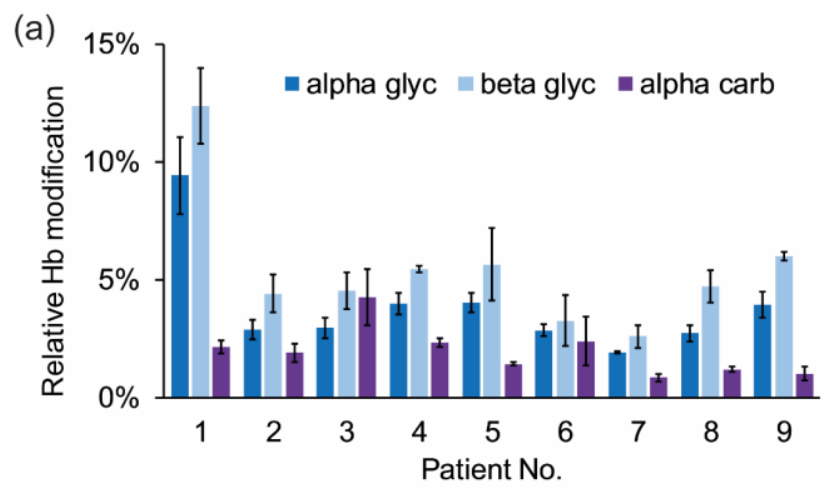

(b)

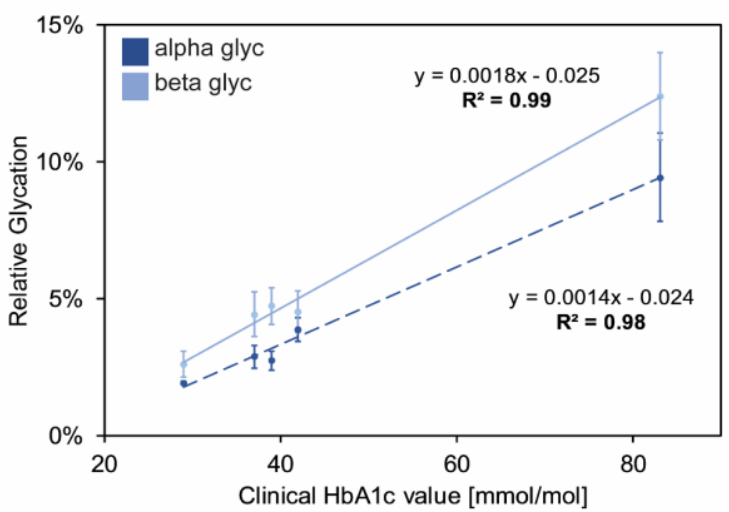

(c)

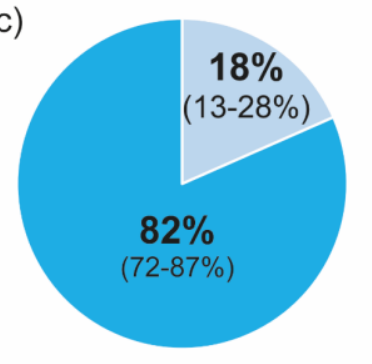

$\begin{array}{ll}\alpha & \text { N-terminal-glycated } \\ & \alpha, \text { lysine-glycated }\end{array}$

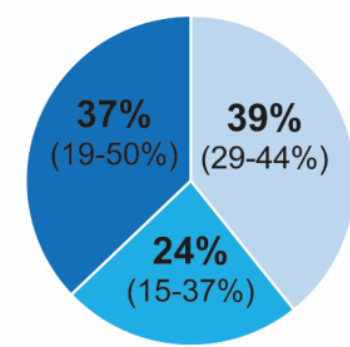

$\mathrm{N}$-terminal-glycated

$\beta$, lysine-glycated (I)

$\beta$, lysine-glycated (II)
Figure 4. Quantification of glycated $\alpha$ - and $\beta-\mathrm{Hb}$, including positional isomers. (a) Relative quantification of glycated $\alpha$ and $\beta-\mathrm{Hb}$ as well as carbamylated $\alpha-\mathrm{Hb}$ by CZE-MS $(\mathrm{n}=3)$ from clinical DBS samples. (b) Correlation between clinical HbA1c values and glycated $\alpha-$ and $\beta$-Hb by CZE-MS. On the $\mathrm{x}$-axis, clinical obtained $\mathrm{HbA} 1 \mathrm{c}$ for each patient are given. The $y$-axis shows the result of relative quantification of the glycated $\alpha$ - and $\beta$-chain compared to the respective unmodified chain. (c) Average ratios of $\mathrm{N}$-terminal- and lysineglycated $\alpha$ - and $\beta-\mathrm{Hb}$ in the clinical samples. The range between individual samples $(n=9)$ is given in brackets. The labels I and II refer to the labels given in Figure $3 \mathrm{~d}$. 
and clinical values (Figure $4 \mathrm{~b}$ ). $\mathrm{R}^{2}$ values close to 1 indicate good linearity between clinical and CZE-MS results, suggesting that the method can be applied for quantification of glycated $\mathrm{Hb}$. The slope for glycated $\beta-\mathrm{Hb}$ is higher than for $\alpha$, which is in accordance with previous results ${ }^{24}$. Figure $4 \mathrm{c}$ shows the average distribution of N-terminal- and lysine-glycated species. Ratios between the individual samples

showed a wide range of values. Interestingly, the sample with the highest overall glycation showed the highest level of $\mathrm{N}$ terminal and lysine-glycation type $\mathrm{I}$ on the $\beta$-chain $(\mathrm{N}$ terminal-glycated: 44\%, lysine-glycated I: 37\%, lysineglycated II: $19 \%$, cp. Figure 4c). Whether these differing ratios are connected to the overall glycation or to any disease state needs to be evaluated in further studies.

Peaks for carbamylated $\alpha-H b$ were identified in all samples, although the extent is low (1-3\%). Carbamylated $\beta-\mathrm{Hb}$ has not been identified. This result is in accordance with in vitro results, indicating that the $\alpha$-chain is also the main carbamylation target in vivo. Based on the verification for glycated $\mathrm{Hb}$ against the clinical routine method, we expect that quantification is also possible for carbamylation and other $\mathrm{Hb}$ proteoforms.

Separation prior to MS detection effectively removes salts and other interfering sample constituencies and helps in overall reduction of sample complexity. Additionally, MS detection offers increased sensitivity compared to optical detection. With our method we are able to quantify glycated and carbamylated $\mathrm{Hb}$-chains and possibly other proteoforms together in a single run at least to the $1 \%$ relative abundance level.

\section{Application to veterinary DBS samples}

Due to sequence differences, routine clinical methods developed for a particular biological species cannot always directly transferred for the analysis of $\mathrm{Hb}$ of different species. To demonstrate versatility and flexibility of our method, we

(a) $2 \mathrm{e}$
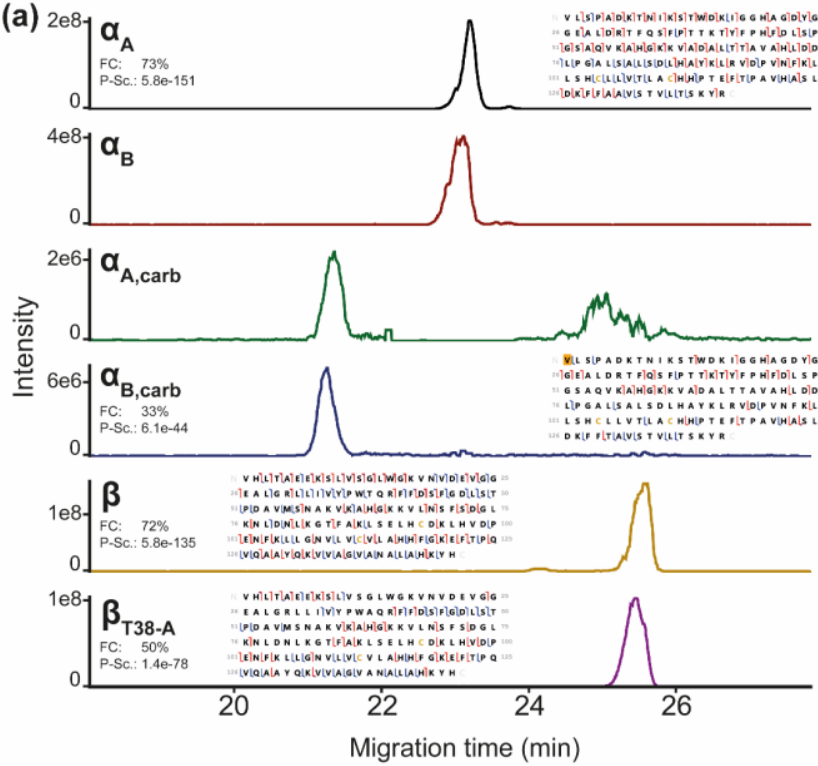

additionally analyzed DBS samples of cats and dogs. Overall, four samples of cats (one healthy, three with chronic kidney disease (CKD) at stages 3-4 according to the International Renal Interest Society (IRIS), http://www.iris-kidney.com) and two healthy dogs were analyzed. Figure 5 shows EIEs of identified $\mathrm{Hb}$-chains and respective proteoforms for dog and cat samples. Interestingly, migration order of $\alpha$ and $\beta$ was reversed in dogs, stressing the need of MS for correct identification of Hb-chains. In the sample of the healthy dogs, a sequence variant $(\mathrm{A} 130 \rightarrow \mathrm{T})$ in the second $\alpha$-chain (referred to as $\alpha_{\mathrm{B}}$ ) of $\mathrm{HbA}$ is known $^{39}$ and was identified. Furthermore, carbamylated $\alpha-\mathrm{Hb}$ was identified in low amounts $(\sim 1 \%)$ for both $\alpha$ - chain variants. An unknown $\beta$-chain variant, with a mass difference of -30 Da has been observed in both dog samples. Intact mass and MS/MS data clearly indicate the species to be a $\mathrm{T} 38 \rightarrow$ A sequence variant (P-score: 1.3e-78). To our knowledge, this variant has not been previously reported.

DBS samples of cats suffering from CKD generally showed presence of various $\mathrm{Hb}$ proteoforms (Figure $5 \mathrm{~b}$ ). The levels of $\alpha$-carbamylation were significantly increased $(\mathrm{p}<0.001, \mathrm{n}=4)$ in the three sick cats $(2.7 \%, 3.0 \%$ and $3.3 \%)$, compared to the healthy individual $(1.1 \%)$. This disease-related increase in carbamylated $\mathrm{Hb}$ has first been reported here for cats and coincides with increased creatinine levels by factors of 2-3 between the CKD cats and the healthy individual. Detailed results can be found in the supporting information.

Additionally, the presence of glutathionylated $\alpha-\mathrm{Hb}$ is suspected based on MS1 data in the three sick cats. Relative amounts were $2.3 \%, 5.6 \%$ and $7.2 \%$ respectively, while it has not been detected in the sample of the healthy cat. Glutathionylation is a hallmark for oxidative stress ${ }^{40}$ and has been connected to uremia in humans ${ }^{41}$. In all of the samples, C-terminal

(b)
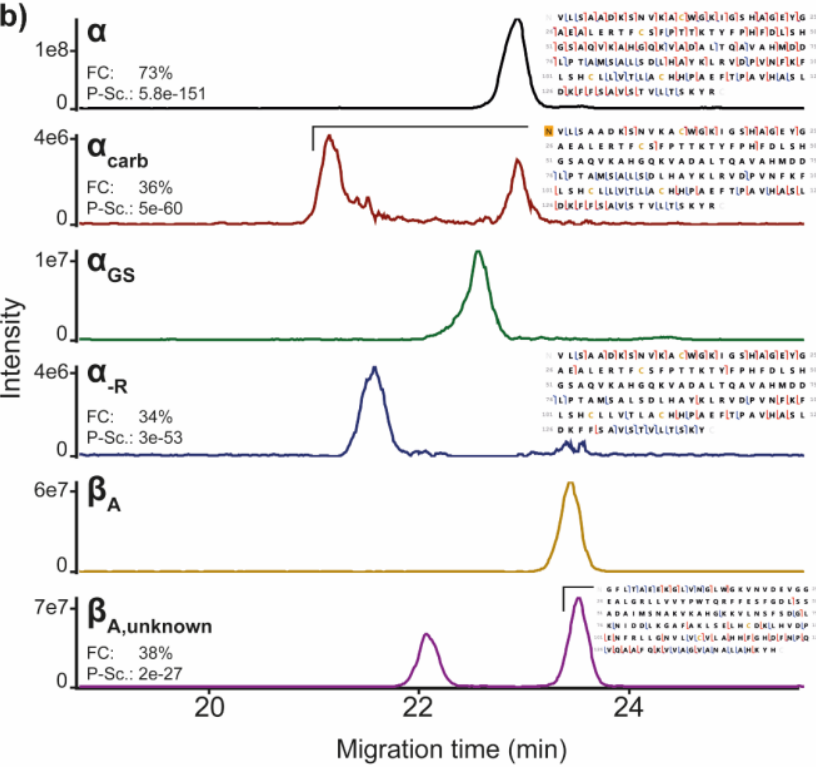

Figure 5. Identified proteoforms from DBS samples of a healthy dog (a) and a cat with CKD (b). All EIEs were created by addition of the three most abundant charge states. Presented proteoforms were identified based on intact mass. For some of the proteoforms, fragment maps, fragmentation coverage and P-scores are given, obtained from additional CZE-MS/MS analysis. 
removal of arginine from the $\alpha$-chain was identified. Carboxypeptidases have been reported to remove C-terminal arginine in human $\alpha-\mathrm{Hb}^{42}$, and these types of enzymes have also been identified in cat ${ }^{43}$. This suggest a similar process of C-terminal arginine removal in cats as has been demonstrated in humans. In the cat sample, an unknown species with an intact mass of $16001 \mathrm{Da}$ has been detected. Top-down data demonstrates that this species is a modified $\beta$-chain or a $\beta$ chain sequence variant. The mass difference to the $\beta$-chain of $\sim 74$ Da suggest a Gly $\rightarrow$ Met substitution. This, however, could not be unambiguously proved.

We want to emphasize here that due to our small dataset we cannot make clear and reliable statements about the clinical significance of our findings. However, especially the elevated levels of carbamylated $\mathrm{Hb}$ in cats with $\mathrm{CKD}$ are consistent with previous pilot data in $\operatorname{dog} s^{5,6}$ and humans $s^{4,7}$. The potential use of carbamylated $\mathrm{Hb}$ as biomarker for $\mathrm{CKD}$ in humans, dogs and cats should be evaluated in further studies. Additionally, these results demonstrate the capabilities of our method to identify unknown or suspected $\mathrm{Hb}$ proteoforms with high confidence

\section{Conclusions}

We have developed a versatile and flexible CZE-top-downMS/MS method for the in-depth characterization of $\mathrm{Hb}$ proteoforms. The applied DEAEDq-PMA coating possesses excellent characteristics for CZE-MS analysis of intact proteins such as reduced protein adsorption, MS-compatibility, moderate EOF and relatively high coating stability. The possibility to fine-adjust the EOF by using different polycations and polyanions for the 5-layers SMIL coating enables high separation power as has been demonstrated by partial separation of positional isomers on intact level. Application of CZE-MS allows identification of known Hb-chains and proteoforms as well as their relative quantification as demonstrated for glycated and carbamylated $\alpha$ - and $\beta-\mathrm{Hb}$. Our quantification results show good correlation with results from a clinical routine method. Quantification of carbamylated $\mathrm{Hb}$ revealed significantly increased levels in cats suffering from CKD compared to a healthy individual. Application of targeted CZE-MS/MS using ETD/HCD methods allows the characterization of known and unknown $\mathrm{Hb}$ proteoforms. The method enabled unambiguous identification and characterization of known $\mathrm{Hb}$ variants in human clinical samples. Moreover, a putative new sequence variant of the $\beta$-chain in dogs was identified. Pseudo SRM enables relative quantification of positional isomers of glycated $\alpha$ - and $\beta-\mathrm{Hb}$. Our data indicates that the $\mathrm{N}$-terminal position represents the main glycation site for $\beta-\mathrm{Hb}$ in vivo, while this is not the case for $\alpha-\mathrm{Hb}$.

The application of DBS offers several advantages such as easy sample taking, long-time sample stability and reduced sample preparation ${ }^{44,45}$-here only a simple extraction step is required. In combination with our top-down approach, omitting the necessity of proteolytic digestion, preparation time and possible artifacts introduction during sample preparation is reduced.

The combination of high separation power, versatility, flexibility and the possibility to obtain information-rich data from a single CZE-MS/MS run makes our screening method an ideal tool to combine with clinical and veterinary routine methods when unknown $\mathrm{Hb}$ proteoforms are suspected or quantification results need to be validated with an orthogonal method. Furthermore, the possibility to separate and characterize positional isomers could be valuable for a deeper understanding of the mechanisms behind non-enzymatic protein modifications. Given the unique properties of $\mathrm{Hb}$ as potential biomarker in various contexts, our method could become very useful for purposes of clinical analysis and general pathophysiological research.

\section{ASSOCIATED CONTENT}

\section{Supporting Information}

The Supporting Information is available free of charge on the ACS Publications website.

Additional experimental details including protocols for in vitro carbamylation and glycation of $\mathrm{Hb}$, capillary conditioning and coating protocols, method details for the $\mathrm{MS}^{2}$ and pseudo SRM method, a list of precursors used for MS/MS experiments, information on the HbAlc determination in the clinic as well as detailed quantification results for carbamylated $\mathrm{Hb}$ in cats.

\section{AUTHOR INFORMATION}

\section{Corresponding Author}

Christian Neusüß - Department of Chemistry, Aalen University, Beethovenstraße 1, 73430 Aalen; Germany

Email: christian.neusuess@hs-aalen.de

\section{Authors}

Alexander Stolz 1) Department of Chemistry, Aalen University, 73430 Aalen, Germany

2) Department of Pharmaceutical and Medicinal Chemistry, Friedrich Schiller University Jena, 07743, Jena, Germany

Ylva Hedeland - Department of Medical Sciences, Clinical Chemistry, Uppsala University Hospital, SE-751 24 Uppsala, Sweden

Liesa Salzer - Department of Chemistry, Aalen University, 73430 Aalen, Germany

$\uparrow$ now at Research Unit Analytical BioGeoChemistry, Helmholtz Center Munich, 85764 Neuherberg, Germany

Jennifer Römer 1) Department of Chemistry, Aalen University, 73430 Aalen, Germany

2) Institute of Analytical Chemistry, Chemo- and Biosensors, University of Regensburg, 93053 Regensburg, Germany

Reidun Heiene - AniCura Animal Hospital, 850462 Oslo, Norway

$\dagger \dagger$ now at Dyreklinikken i Bamble, 3960 Stathelle., Norway

Laurent Leclercq - IBMM, University of Montpellier, CNRS, ENSCM, 34090 Montpellier, France

Hervé Cottet - IBMM, University of Montpellier, CNRS, ENSCM, 34090 Montpellier, France

Jonas Bergquist - Department of Chemistry - Biomedical Center, Analytical Chemistry, Uppsala University, Box 599, SE-751 24 Uppsala, Sweden

Notes

The authors declare no conflict of interest.

\section{ACKNOWLEDGMENT}

The authors want to thank François de l'Escaille for providing the prototype interface for CE-MS coupling to the Orbitrap MS and Víctor González-Ruiz for technical support with the interface. 
Furthermore, the authors want to thank Kevin Jooß and John P. McGee for valuable discussions and support with data interpretation. Additional thanks goes to the Swedish research council grant No. 2015-4870.

\section{REFERENCES}

(1) Patrinos, G. P.; Giardine, B.; Riemer, C.; Miller, W.; Chui, D. H. K.; Anagnou, N. P.; Wajcman, H.; Hardison, R. C. Nucleic Acids Res. 2004, 32 (1), 537-41.

(2) Weatherall, D. J. Curr. Mol. Med. 2008, 8 (7), 592-599.

(3) Franco, R. S. Transfus. Med. Hemother. 2012, 39 (5), 302-307.

(4) Davenport, A.; Jones, S.; Astley, J. P.; Hartog, M. Lancet 1993, 341 (8861), 1614-1617.

(5) Heiene, R.; Vulliet, P. R.; Williams, R. L.; Cowgill, L. D. Am. J. Vet. Res. 2001, 62 (8), 1302-1306.

(6) Vaden, S. L.; Gookin, J.; Trogdon, M.; Langston, C. E.; Levine, J.; Cowgill, L. D. Am. J. Vet. Res. 1997, 58 (11), 1193-1196. (7) Davenport, A.; Jones, S.; Goel, S.; Astley, J. P.; Feest, T. G. Kidney Int. 1996, 50 (4), 1344-1351.

(8) Edelman, D.; Olsen, M. K.; Dudley, T. K.; Harris, A. C.; Oddone, E. Z. J. Gen. Intern. Med. 2004, 19 (12), 1175-1180.

(9) Negre-Salvayre, A.; Salvayre, R.; Augé, N.; Pamplona, R.;

Portero-Otín, M. Antioxid. Redox Signal. 2009, 11 (12), 3071-3109. (10) Hoelzel, W.; Miedema, K. J. Int. Fed. Clin. Chem. 1996, 8 (2), 62-4, 66-7.

(11) Hoelzel, W.; Weykamp, C.; Jeppsson, J.-O.; Miedema, K.; Barr, J. R.; Goodall, I.; Hoshino, T.; John, W. G.; Kobold, U.; Little, R.; Mosca, A.; Mauri, P.; Paroni, R.; Susanto, F.; Takei, I.; Thienpont, L.; Umemoto, M.; Wiedmeyer, H.-M. Clin. Chem. 2004, 50 (1), 166174

(12) UK Prospective Diabetes Study (UKPDS) Group Lancet 1998, 352 (9131), 837-853.

(13) Weykamp, C. Ann. Lab. Med. 2013, 33 (6), 393-400.

(14) Smith, L. M.; Kelleher, N. L. Nat. Methods 2013, 10 (3), 186187.

(15) Bisse, E.; Zorn, N.; Eigel, A.; Lizama, M.; Huaman-Guillen, P.; Marz, W.; van Dorsselaer, A.; Wieland, H. Clin. Chem. 1998, 44 (10), 2172-2177.

(16) Bry, L.; Chen, P. C.; Sacks, D. B. Clin. Chem. 2001, 47 (2), 153 163.

(17) Chen, D.; Crimmins, D. L.; Fong, H. F.; Lindberg, F. P.; Scott, M. G. Clin. Chem. 1998, 44 (6), 1296-1301.

(18) Kabytaev, K.; Connolly, S.; Rohlfing, C. L.; Sacks, D. B.; Stoyanov, A. V.; Little, R. R. Clin. Chim. Acta. 2016, 458, 40-43. (19) Lapolla, A.; Molin, L.; Traldi, P. Int. J. Endocrinol. 2013, 2013 , 412103

(20) Roberts, N. B.; Amara, A. B.; Morris, M.; Green, B. N. Clin.

Chem. 2001, 47 (2), 316-321.

Insert Table of Contents artwork here

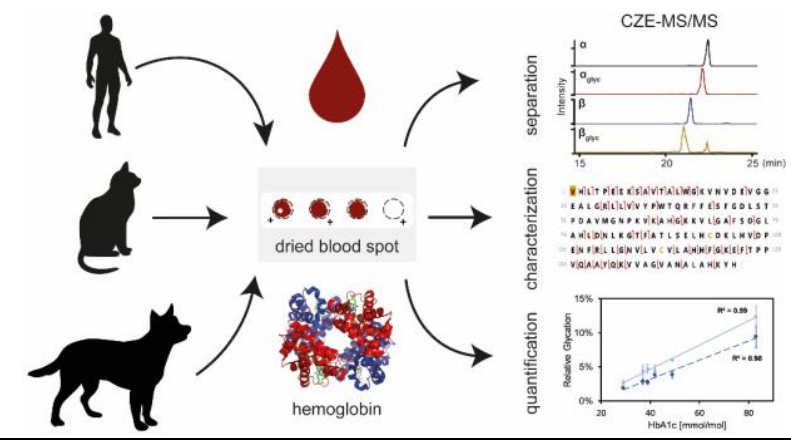

(21) Roberts, N. B.; Green, B. N.; Morris, M. Clin. Chem. 1997, 43 (5), 771-778

(22) Hofstadler, S. A.; Severs, J. C.; Smith, R. D.; Swanek, F. D.; Ewing, A. G. Rapid Commun. Mass. Spectrom. 1996, 10 (8), 919922.

(23) Nguyen, A.; Moini, M. Anal. Chem. 2008, 80 (18), 7169-7173.

(24) Redman, E. A.; Ramos-Payan, M.; Mellors, J. S.; Ramsey, J. M. Anal. Chem. 2016, 88 (10), 5324-5330.

(25) Huhn, C.; Ramautar, R.; Wuhrer, M.; Somsen, G. W. Anal. Bioanal. Chem. 2010, 396 (1), 297-314.

(26) Bekri, S.; Leclercq, L.; Cottet, H. J. Chromatogr. A 2015, 1399, $80-87$.

(27) Leclercq, L.; Morvan, M.; Koch, J.; Neusüß, C.; Cottet, H. Anal. Chim. Acta. 2019, 1057, 152-161.

(28) Demirev, P. A. Anal. Chem. 2013, 85 (2), 779-789.

(29) Jesús, V. R. de; Mei, J. V.; Bell, C. J.; Hannon, W. H. Semin. Perinatol. 2010, 34 (2), 125-133.

(30) Edwards, R. L.; Martin, N. J.; Cooper, H. J. Bioanalysis 2013, 5 (16), 2043-2052.

(31) Bae, J. W.; Lee, M. H. J. Ethnopharmacol. 2004, 91 (1), 137 140 .

(32) Weykamp, C. W.; Penders, T. J.; Siebelder, C. W.; Muskiet, F. A.; van der Slik, W. Clin. Chem. 1993, 39 (1), 138-142.

(33) González-Ruiz, V.; Codesido, S.; Far, J.; Rudaz, S.; Schappler, J. Electrophoresis 2016, 37 (7-8), 936-946.

(34) Thein, S. L.; Menzel, S. Br. J. Haematol. 2009, 145 (4), 455467.

(35) Davison, A. S.; Green, B. N.; Roberts, N. B. Clin. Chem. Lab. Med. 2008, 46 (9), 1230-1238.

(36) Hao, P.; Ren, Y.; Alpert, A. J.; Sze, S. K. Mol. Cell. Proteomics 2011, 10 (10), O111.009381.

(37) Krokhin, O. V.; Antonovici, M.; Ens, W.; Wilkins, J. A.; Standing, K. G. Anal. Chem. 2006, 78 (18), 6645-6650.

(38) Thornalley, P. J.; Rabbani, N. Biochim. Biophys. Acta 2014, 1840 (2), 818-829.

(39) Brimhall, B.; Duerst, M.; Jones, R. T. J. Mol. Evol. 1977, 9 (3), 231-235.

(40) Niwa, T. J. Chromatogr. B Analyt. Technol. Biomed. Life Sci. 2007, 855 (1), 59-65.

(41) Fumio Takayama; Shuichi Tsutsui; Masanobu Horie; Kaoru Shimokata; Toshimitsu Niwa Kidney Int. Suppl. 2001, 78, 155-158. (42) Michel, B.; Igić, R.; Leray, V.; Deddish, P. A.; Erdös, E. G. Circ. Res. 1996, 78 (4), 635-642.

(43) Vernigora, A. N.; Gengin, M. T. Biochemistry Mosc. 2003, 68 (1), 80-85.

(44) Sharma, A.; Jaiswal, S.; Shukla, M.; Lal, J. Drug. Test. Anal. 2014, 6 (5), 399-414.

(45) Wagner, M.; Tonoli, D.; Varesio, E.; Hopfgartner, G. Mass. Spectrom. Rev. 2016, 35 (3), 361-438. 